\title{
A systematic review on improving the biocompatibility of titanium implants using nanoparticles
}

\author{
Nthabiseng Nhlapo ${ }^{1}$, Thywill Cephas Dzogbewu ${ }^{1, *}$, and Olga de Smidt ${ }^{2}$ \\ ${ }^{1}$ Department of Mechanical and Mechatronics Engineering, Central University of Technology, Free State, Private Bag X 20539, \\ Bloemfontein, Free State, South Africa \\ 2 Department of Life Sciences, Central University of Technology, Free State, Private Bag X 20539, Bloemfontein, Free State, \\ South Africa
}

Received: 13 July 2020 / Accepted: 30 August 2020

\begin{abstract}
An ideal biomaterial should be biointegratable with minimum adverse immune response. Titanium (Ti) and its alloys are widely used biomaterials for manufacturing clinical implants because of their innate biocompatibility. However, the bioinert property of Ti may hinder tissue-implant integration and its biocompatibility nature allows for attachment of bacterial cells on implant surfaces. Nanoparticles (NPs) have been proposed as a possible intervention to overcome these biological shortcomings of Ti-based implants. The aim of the current systematic review was to identify literature that demonstrates enhanced biocompatibility of Ti-based implants by incorporating NPs. Electronic searches were conducted through the PubMed/MEDLINE, ScienceDirect, Web of Science and EBSCOhost databases. Studies published in English were extracted, without restrictions on the year of publication, using the following keywords: 'biocompatibility', 'nanoparticles', 'titanium' and 'implant'. The guidelines stipulated in the Preferred Reporting Items for Systematic Reviews and Meta-Analyses (PRISMA) Statement were followed. A total of 630 articles were identified in the initial search and upon reviewing, 21 articles were selected according to the eligibility criteria. The selected literature showed robust evidence to support the hypothesis that the inclusion of NPs improves biocompatibility of Ti implants. The studies further indicated a close correlation between biocompatibility and antibacterial properties, of which NPs have been proven to characteristically achieve both.
\end{abstract}

Keywords: Antibacterial / biocompatibility / cytotoxicity / nanoparticles / titanium-based implants

\section{Introduction}

Biomaterial are typically expected to be integratable with the biological system and stimulate minimum adverse tissue response $[1,2]$. Titanium $(\mathrm{Ti})$ and its alloys are widely used in medicine and dentistry because they exhibit minimal immunogenic potential in vivo and display superior biostability and biocompatibility in comparison to allogenic grafts and other biomaterial [3-5]. In contrast, $\mathrm{Ti}$ is a bioinert metal and thus does not initiate the initial cellular responses required to achieve biocompatibility [6]. This may subsequently interfere with bone repair at the tissue-implant interface and result in implantation failure. Incorporating nanoparticles (NPs) has emerged as an effective intervention towards improving the biological principles of Ti-based implants.

\footnotetext{
* e-mail: thydzo@yahoo.fr
}

The synergy of biointegration without triggering an immune response is achievable through the use of foreign material whose properties resemble those of the innate tissue [5]. Nano-sized particles are structurally similar to various body proteins, ligands receptors and deoxyribonucleic acid (DNA) [1] Moreover, nanobiomaterials have been established as able to absorb living cells and, therefore, may be utilized to transport nucleic acids and conjugate with organic material, which contributes significantly to biointegration [7]. NPs include ultrafine structures with nano-range dimensions in diameter and are composed of any type of biocompatible substance [8,9]. It is well documented that incorporating nano-sized particles increases the biocompatibility and bioactivity of implant materials more efficiently as compared to similar bulk material $[6,9,10]$.

The probability of implant success may be further enhanced by coating the Ti-based implants with material that exhibits both biocompatibility and antimicrobial activity; particularly since Ti surfaces not only promote 
cell adhesion followed by osseointegration, but also promote bacterial adhesion resulting in infection and inflammatory processes $[4,6,9]$. Inorganic NPs are hydrophilic, biocompatible, highly stable, have a low toxicity profile and are not readily susceptible to microbial attack [7]. Enriching Ti implants with NPs, such as copper, silver and zinc, allows for antimicrobial efficacy and noncytotoxicity against human cells [5,11]. The superior antibacterial properties of metal NPs hinder bacterial attachment and present further beneficial clinical treatments since these assembling NPs may prevent infection during the bone healing process $[4,9]$.

Technologies involving nanoparticle-based methods for delivery of bioactive molecules and enhancing biocompatibility and cellular survival continue to evolve with the intended use being for the clinical environment [10]. The prescribed sequence of biological research requires for the implant material to first undergo in vitro cytotoxicity testing procedures, followed by in vivo testing to describe the interaction with soft tissue and assess the biocompatibility of these devices [5,12]. However, the detailed cellular mechanisms at the boneimplant interface during osteogenesis and the actual mechanism of interaction of NPs with different cells in a biological medium are still largely unknown [13]. Furthermore, the extent to which modifying $\mathrm{Ti}$ implants to include NPs enhances the biocompatibility of these implants is not well documented. Therefore, the aim of the current systematic review was to establish evidence which support the hypothesis that NPs improve the biocompatibility properties of Ti-based implants while prolonging the antibacterical effects.

\section{Materials and methods}

\subsection{Review question}

The addressed focus question was 'Does the inclusion of nanoparticles in manufacturing titanium-based implants enhance biocompatibility of the implants?' and developed according to the Participant, Intervention, Control, Outcome (PICO) principle as follows [14]:

(P) Participants: The test samples were intended for human implantation treatment and should be Ti-based.

(I) Types of interventions: The interventions of interest were those demonstrating enhancement of biocompatibility by using NPs. NPs.

(C) Control intervention: Ti implant material without

(O) Outcome measures: Improved biocompatibility and reduced bacterial infection in samples with NPs.

\subsection{Procedure}

The present systematic review was conducted according to the guidelines set out in the Preferred Reporting Items for Systematic Reviews and Meta-Analyses (PRISMA) Statement [15]. The studies were selected according to the eligibility criteria described in the proceeding subsection.

\subsection{Eligibility criteria}

The extracted articles contained data indicating the extend to which NPs affect biocompatibility of Ti implants. In vitro and in vivo studies were considered with the implant material intended for human use. Furthermore, the inclusion criteria consisted of studies published in English and without restrictions on the date range of publication. The studies that were excluded were those that did not use $\mathrm{Ti}$ as the base biomaterial, attributed biocompatibility to the use of micro/macro particles or nano-forms other than NPs and those that assumed biocompatibility without evaluations thereof.

\subsection{Information sources}

Information was retrieved from the following electronic databases that contain articles published in journals relating to biomedical sciences: PubMed/MEDLINE, ScienceDirect, Web of Science and EBSCOhost. Additional sources were used to supplement the data obtained.

\subsection{Search strategy}

The selected electronic databases were searched using the keywords 'biocompatibility', 'nanoparticles', 'titanium' and 'implant'. These keywords were applied to the databases as follows:

- For PubMed/MEDLINE, the keywords were entered as (biocompatibility AND nanoparticles AND titanium AND implant).

- For ScienceDirect, the keywords were entered as ('biocompatibility' AND 'titanium' AND 'implant' AND 'nanoparticles').

- For Web of Science, the topic searches were entered as (biocompatibility* AND titanium AND implant* AND (nanoparticle* OR nano particle*)).

- For EBSCOHost, the Boolean search phrase was (biocompatibility AND titanium AND implant AND nanoparticles).

The full-texts were downloaded either directly via the links provided on the databases or via ResearchGate and Google Scholar profiles for the articles identified using additional sources.

\subsection{Criteria for studies selection}

Data selection began with analysing the titles followed by carefully evaluating the abstracts of the titles indicating inclusion. Thereafter, the articles that were considered eligible for review were selected for reading of the fulltexts. Two reviewers to ascertain whether the studies fulfilled the inclusion criteria thus independently reviewed the obtained full-texts of the studies. Inconclusive decisions among the researchers were resolved by consulting the third reviewer. Studies that were unanimously selected as fulfilling the inclusion criteria were processed for data extraction. 


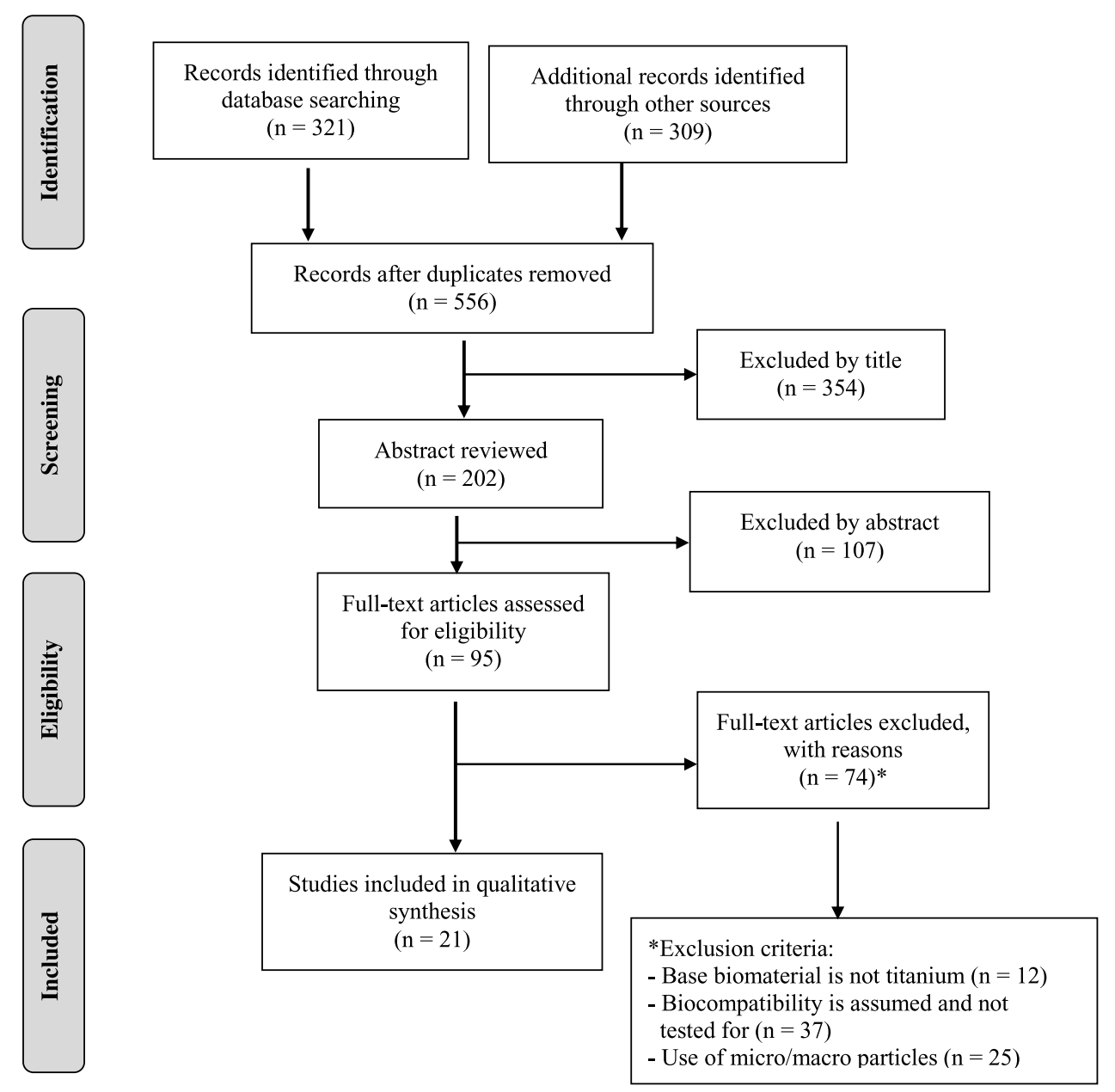

Fig. 1. The PRISMA flow chart for search strategy and selection criteria for eligible articles.

\subsection{Risk of bias assessment}

The Cochrane Collaboration's tool for assessing risk of bias was used to assess the individual studies [16]. The risk of bias assessment parameters were as following:

- Random sequence generation $(\sqrt{ } / \times / ?)$;

- Allocation concealment $(\sqrt{ } / \times / ?)$;

- Blinding of participant's and personnel $(\sqrt{ } / \times / ?)$;

- Blinding of outcomes assessment $(\sqrt{ } / \times / ?)$;

- Incomplete outcome data $(\sqrt{ } / \times / ?)$;

- Selective reporting $(\sqrt{ } / \times / ?)$;

- Other sources of bias $(\sqrt{ } / \times / ?)$.

\subsection{Additional analyses}

Antibacterial examinations were considered since $\mathrm{Ti}$ significantly lacks in antibacterial properties and exhibits susceptibility to bacterial colonisation, which may negatively affect the biocompatibility functions of $\mathrm{Ti}$ implant. Additionally, nanoparticles are renowned antibacterial agents.

\section{Results}

\subsection{Study selection}

The flowchart of the systematic review is depicted according to the PRISMA flow diagram in Figure 1. Searches of the selected databases retrieved 321 articles and were supplemented with 309 articles from additional sources, which resulted in a total of 630 titles identified in the initial search. After the duplicate records were removed, 556 articles remained. A further 354 and 107 articles were excluded after screening the titles and the abstracts, respectively. Eligibility of the full-texts of the articles was assessed from which 74 article were found to not fulfil the inclusion criteria. Finally, 21 articles were included in the present systematic review and processed for data extraction in the following order (Tab. 1): substrate material, type of nanoparticles (diameter in $\mathrm{nm}$ ), type of study, type of cell/organism, type of laboratorial analysis, type of biocompatibility test, experimental/ incubation period, was antibacterial effect tested for? and references. 


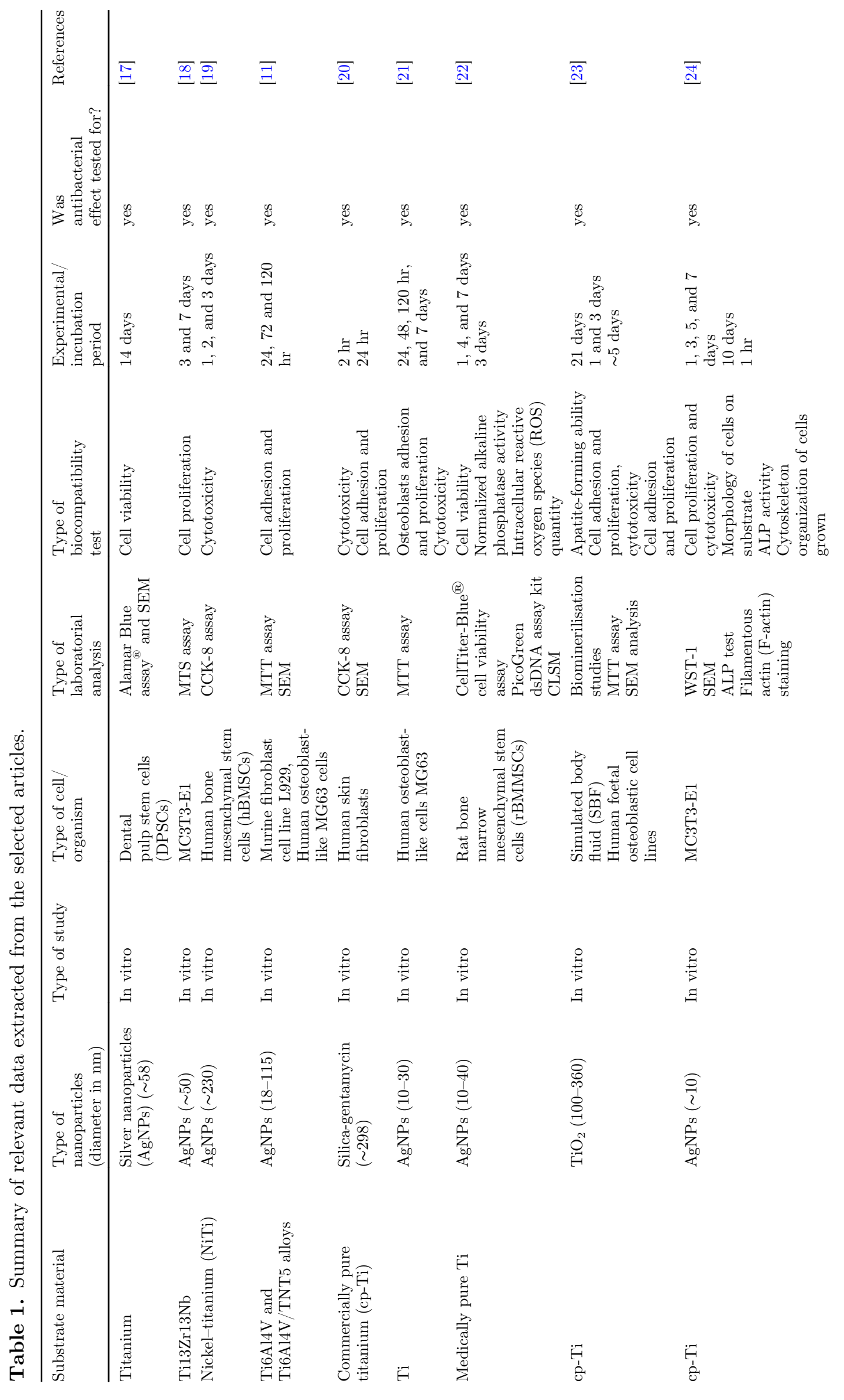




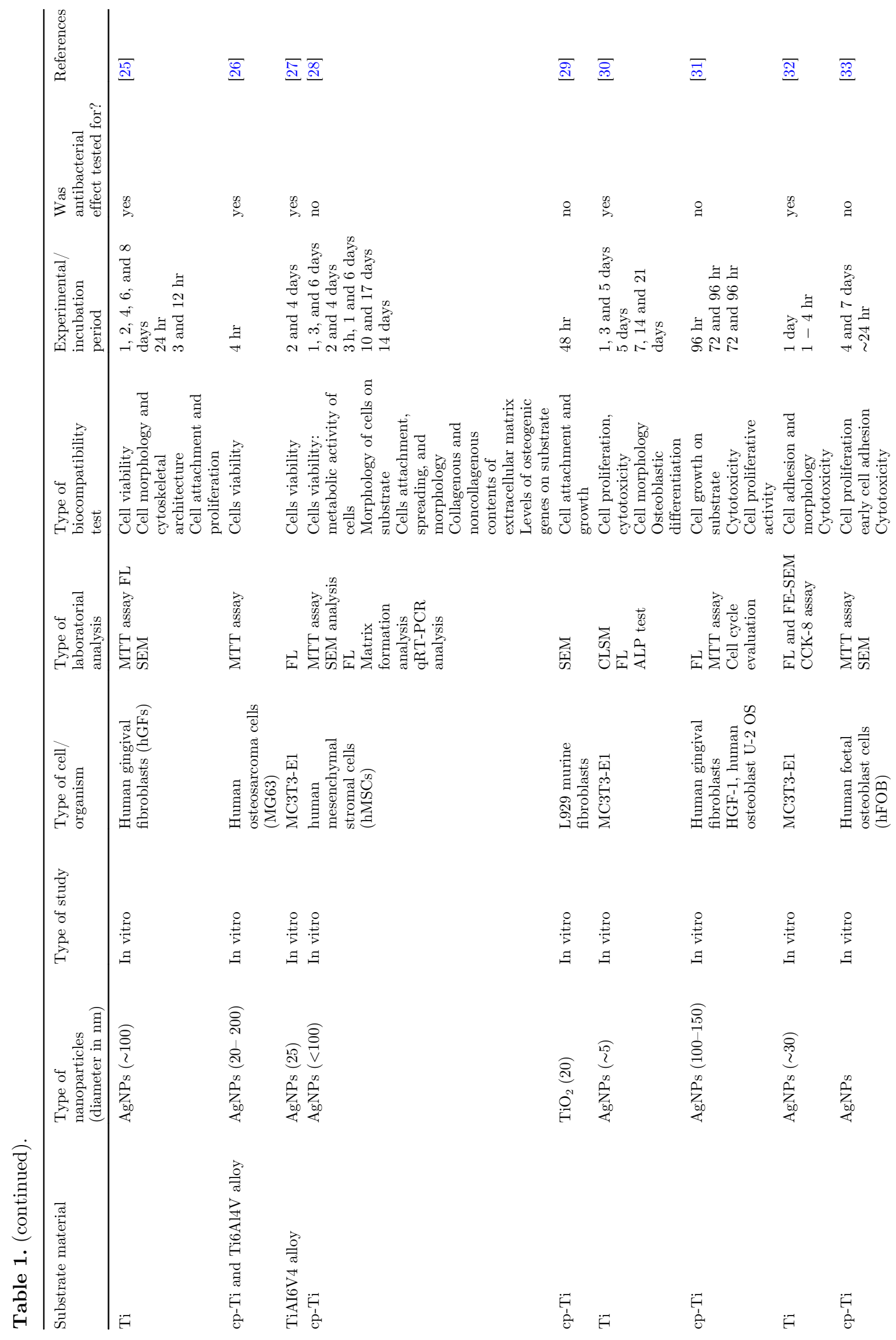




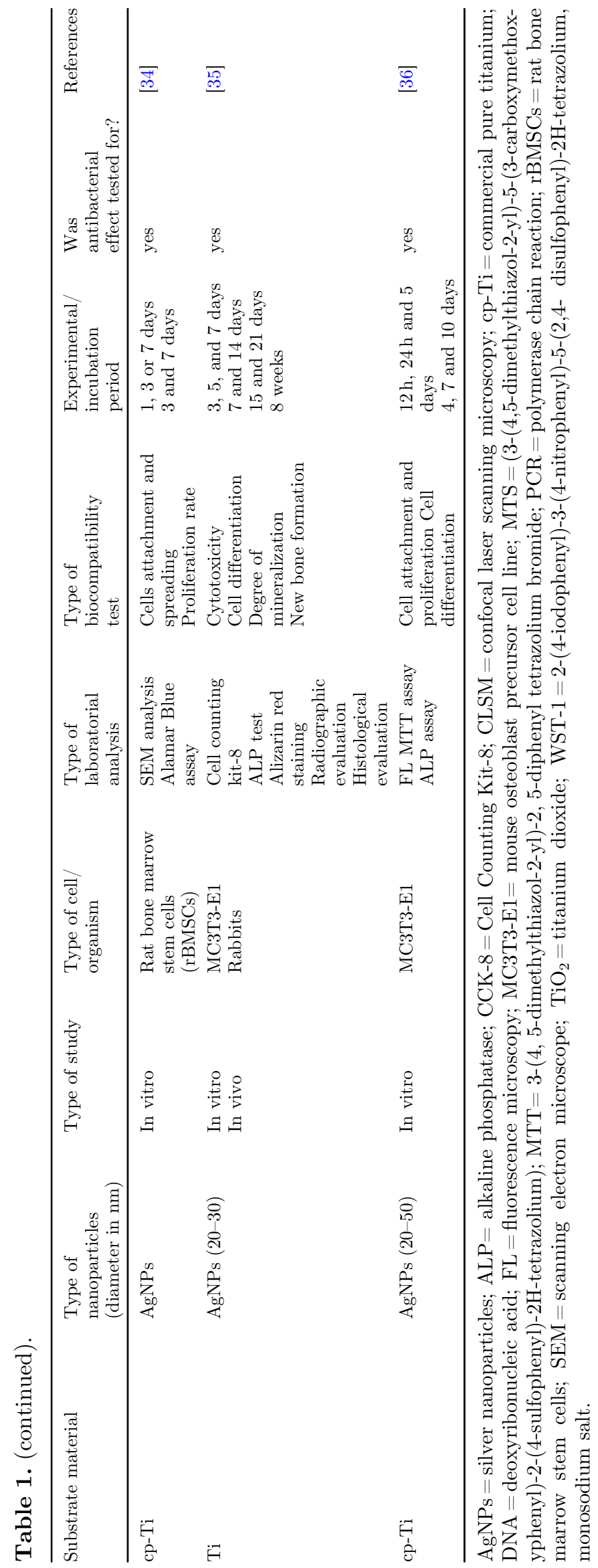


Table 2. Risk of bias assessment summary of the seleceted studies.

\begin{tabular}{|c|c|c|c|c|c|c|c|}
\hline & $\begin{array}{l}\text { Random } \\
\text { sequence } \\
\text { generation }\end{array}$ & $\begin{array}{l}\text { Allocation } \\
\text { concealment }\end{array}$ & $\begin{array}{l}\text { Blinding of } \\
\text { participants } \\
\text { and personnel }\end{array}$ & $\begin{array}{l}\text { Blinding of } \\
\text { outcomes } \\
\text { assessment }\end{array}$ & $\begin{array}{l}\text { Incomplete } \\
\text { outcome } \\
\text { data }\end{array}$ & $\begin{array}{l}\text { Selective } \\
\text { reporting }\end{array}$ & $\begin{array}{l}\text { Other } \\
\text { sources } \\
\text { of bias }\end{array}$ \\
\hline [17] & $\times$ & $x$ & $?$ & $\sqrt{ }$ & $\sqrt{ }$ & $\sqrt{ }$ & $?$ \\
\hline [18] & $x$ & $x$ & $?$ & $\sqrt{ }$ & $\sqrt{ }$ & $\sqrt{ }$ & $?$ \\
\hline [19] & $\times$ & $\times$ & $?$ & $\sqrt{ }$ & $\times$ & $\sqrt{ }$ & $?$ \\
\hline [11] & $\times$ & $x$ & $?$ & $\sqrt{ }$ & $\sqrt{ }$ & $\sqrt{ }$ & $?$ \\
\hline [20] & $\times$ & $x$ & $?$ & $\sqrt{ }$ & $\sqrt{ }$ & $\sqrt{ }$ & $?$ \\
\hline [21] & $x$ & $x$ & $?$ & $\sqrt{ }$ & $\sqrt{ }$ & $\sqrt{ }$ & $\times$ \\
\hline [22] & $x$ & $x$ & $?$ & $\sqrt{ }$ & $\sqrt{ }$ & $\sqrt{ }$ & $\sqrt{ }$ \\
\hline [23] & $\times$ & $x$ & $?$ & $\sqrt{ }$ & $\times$ & $x$ & $x$ \\
\hline [24] & $x$ & $x$ & $?$ & $\sqrt{ }$ & $\sqrt{ }$ & $\sqrt{ }$ & $\sqrt{ }$ \\
\hline [25] & $x$ & $x$ & $?$ & $\sqrt{ }$ & $\sqrt{ }$ & $\sqrt{ }$ & $?$ \\
\hline [26] & $\times$ & $\times$ & $?$ & $\sqrt{ }$ & $\sqrt{ }$ & $\sqrt{ }$ & $?$ \\
\hline [27] & $x$ & $x$ & $?$ & $\sqrt{ }$ & $\times$ & $\times$ & $\times$ \\
\hline [28] & $\times$ & $x$ & $?$ & $\sqrt{ }$ & $\sqrt{ }$ & $\sqrt{ }$ & $\sqrt{ }$ \\
\hline [29] & $x$ & $x$ & $?$ & $\sqrt{ }$ & $\times$ & $x$ & $\times$ \\
\hline [30] & $\times$ & $x$ & $?$ & $\sqrt{ }$ & $\sqrt{ }$ & $\sqrt{ }$ & $\times$ \\
\hline [31] & $\sqrt{ }$ & $\sqrt{ }$ & $\sqrt{ }$ & $\sqrt{ }$ & $\sqrt{ }$ & $\sqrt{ }$ & $x$ \\
\hline [32] & $\times$ & $\times$ & $?$ & $\sqrt{ }$ & $\sqrt{ }$ & $\sqrt{ }$ & $?$ \\
\hline [33] & $x$ & $x$ & $?$ & $\sqrt{ }$ & $\sqrt{ }$ & $\sqrt{ }$ & $?$ \\
\hline [34] & $\sqrt{ }$ & $\sqrt{ }$ & $\sqrt{ }$ & $\sqrt{ }$ & $\sqrt{ }$ & $\sqrt{ }$ & $?$ \\
\hline [35] & $\times$ & $x$ & $?$ & $\sqrt{ }$ & $\sqrt{ }$ & $\sqrt{ }$ & $?$ \\
\hline [36] & $\times$ & $\times$ & $?$ & $\sqrt{ }$ & $\sqrt{ }$ & $\times$ & $?$ \\
\hline
\end{tabular}

$\sqrt{ }=$ low risk; $?=$ unclear risk; $\times=$ high risk.

\subsection{Risk of bias assessment}

The results from the risk of bias assessment are summarised in Table 2. The results showed that 16 studies were considered to have a low risk of bias while three were considered to have high risk of bias since the authors had opted not to include all the initially prepared test samples in all the analyses. The remaining two were considered to be of moderate risk.

\subsection{Study characteristics}

From Table 1, it may be observed that of the 21 studies included in this systematic review, $18(86 \%)$ made use of silver nanoparticles (AgNPs) with the diameters ranging between 10 and $230 \mathrm{~nm}$. Other nanoparticle materials used were titanium dioxide $\left(\mathrm{TiO}_{2}\right)$ and silica-gentamycin with particle diameters of between 20 and $360 \mathrm{~nm}$. The NPs were added to the substrates by means of surface modification techniques that include depositing the particles onto the surface or incorporating the NPs in a coating solution.

Common methods of analyses were 3-(4,5-dimethylthiazol-2-yl)-2,5-diphenyl tetrazolium bromide (MTT) assay, Cell Counting Kit-8 (CCK-8) assay, alkaline phosphatase (ALP) test and surface morphology characterisation using a scanning electron microscope (SEM) and a fluorescence microscope. These techniques were mainly applied to determine biocompatibility of the NPs in terms of adhesion and proliferation of cells on the surfaces of the substrates (MMT assay and microscopic observations), cytotoxicity of the nanoparticle (MMT and CCK-8 assays) and cell differentiation (ALP test).

Majority of the studies (95\%) were conducted in vitro and mainly against cultured human osteoblast and fibroblast cell lines (48\%) as well as cultured mouse osteoblast cell line (MC3T3-E1) (33\%). [35] conducted in vivo analyses to qualify biocompatibility and biocidal aspects using rabbits while [32] performed in vivo antibacterial assay using male rats. A total of 17 (81\%) studies examined the antimicrobial ability of NPs along with the biocompatibility evaluations. Most of the antibacterial testing was carried out on the common Gram-positive Staphylococcus aureus (S. aureus) and Gram-negative Escherichia coli (E. coli) bacteria.

\section{Discussion}

Nanomaterials have been deemed as the 'material of the 21st century' because of their unique designs and wider combination of properties as compared with conventional materials [37]. In addition, there is growing interest in the application of nanoparticles to control various infections because of their biocidal properties and anti-adhesive 
capabilities against biofilms, particularly since biofilm formation on the surface of implant devices may cause periimplantitis and lead to bone loss [25,38]. However, the extensive use of NPs, especially in medicine, incites the consideration of the potential toxicity to the human body. Therefore, along with cell adhesion and proliferation, cytotoxic potential of NPs is one of the most common features investigated during in vitro studies to determine biocompatibility [39]. These biocompatibility features were included in the studies selected in the present systematic review.

The results of the cell viability assay conducted by [17] illustrated that AgNPs implanted titanium surface favoured biocompatibility for long-term use whereby the surface treated with NPs showed unchanged cell viability as compared to the surface control. The authors also evaluated the antibacterial properties of the prepared surfaces against $S$. aureus and AgNPs containing surfaces exhibited a distinct inhibition of bacterial growth, while the nanoparticle deficient surfaces showed minimal effect on bacterial growth. These results are in agreement with those observed by [18] and [19] which showed that modifying Ti specimen surfaces with nanosilver does not deteriorate the overall biocompatibility of the material construct while allowing for adequate antibacterial protection.

During the study of [11], the lowest addition of NPs revealed optimal biointegration properties and high biocidal properties. The MTT assay and SEM micrographs of the tested samples showed an increase in the fibroblast and osteoblast proliferation over time. The viability of the cells after 120 hours of incubation was $85 \%$ or more as compared to reference specimens and thus demonstrated reasonable biocompatibility of the tested nanomaterials. The MTT test of [21] showed that on AgNP-modified coating, there was an initial reduction in human osteoblast cell-like MG63 cell viability at 24 hours followed by an increase after seven days of culture and reached a mean value higher than $60 \%$ over the control culture. An antimicrobial effect was demonstrated and ascribed to the presence of NPs.

[23] and [24] revealed that low concentrations of NPs are favourable for preosteoblast spreading and cytotoxicity prevention. [23] further showed through the MTT assay that the addition of $\mathrm{TiO}_{2} \mathrm{NPs}$ at low concentration (2 and $5 \mathrm{wt} . \%)$ to polymetric scaffolds advanced the proliferation of human foetal osteoblast cells (hFOB) cells. SEM analysis showed higher proliferation of hFOB cells on the scaffolds containing $\mathrm{TiO}_{2} \mathrm{NPs}$ after only three days of culture. The added $\mathrm{TiO}_{2}$ NPs increase the surface area and surface hydrophilicity, thus supporting cell adhesion on the first day and cell proliferation on the third day. Optimized nanoparticle concentration ( $\sim 5 \mathrm{wt} . \%)$ may be applied to engineering scaffolds favouring new tissue formation [23].

[24] discovered that at extended incubation periods, cell extensively spread and secreted abundant extracellular matrix to benefit cell proliferation for AgNP concentrations of $0.01,0.001$, and $0.0001 \mathrm{M}$. Furthermore, low concentrations of AgNPs exhibited no obvious negative effects on the osteogenic differentiation of the MC3T3-E1 after 10 days of culture during the ALP assay. The ALP activity was measured as an early marker for osteogenic differentiation potential of preosteoblasts. Also, substrate groups coated with AgNPs showed significant antibacterial effects against $S$. aureus and $E$. coli. The antimicrobial activities were positively correlated with the nanoparticle dosage loaded on surfaces of substrates. In support of these findings, the microscopic analysis and CCK- 8 test conducted by [20] revealed good biocompatibility properties of all tested specimens where the released silicagentamycin NPs was biocompatible with human fibroblasts. In addition, after incorporating NPs, an effective antibacterial coating on the Ti substrate was achieved.

The unambiguous biocompatibility and antibacterial effects of NPs was further demonstrated by [35] during their in vitro and in vivo investigations. In vitro, the nanosilver-coated Ti samples promoted the ALP activity of MC3T3-E1 cells more effectively with a significant increase observed for extended incubation periods. Moreover, matrix mineralisation of cells (which was observed after 21 days culture in osteogenic medium) on the NPs sample was 2.15 times greater than that on the uncoated Ti control and the CCK-8 assay showed that the NPs did not reduce MC3T3-E1 cell growth, but improved cell proliferation more effectively. The in vitro antibacterial experiments indicated that the uncoated $\mathrm{Ti}$ control did not inhibit bacterial adhesion or proliferation, while nanosilverconcentration-dependent animicrobial behaviour was observed in all nanoparticle-embedded surfaces. These results indicating cell-substrate compatibility and biocidal impact of nanoparticle coated samples were supported by the in vivo studies of [35] in the tibial canals of rabbits. During these studies, specimens with AgNPs accelerated the formation of new bone while suppressing survival of methicillin-resistant $S$. aureus and the Pseudomonas aeruginosa. Therefore, implants impregnated with NPs have simultaneous antibacterial and osteoinductive activities in vitro and in vivo proportional to the concentration of NPs [35]. The in vivo antibacterial assay of [32] showed similar results of the long-term antibacterial ability of $\mathrm{Ti}$ substrates coated with AgNPs.

Applications of NPs in the selected research papers was mainly in the form of embedding the ultrafine structures into a coating material such as nanosilver-loaded bone cement coatings [18], nanosilver-loaded dopamine coatings [36], AgNP-filled hydrogen titanate nanotube layer [30], Ag nanoparticle-loaded $\mathrm{TiO}_{2}$ nanorods (NRDs) coatings [32], hydroxyapatite (HA) coatings [34], thin mussel adhesive protein (Mefp-1)/AgNP composite film [24] and crosslinked gelatin/SG composite coating [20]. The incorporation of NPs into such surface modification coatings facilitates the slow release of ions from NPs and the subsequent reduction of toxicity and prolonged antibacterial effects.

\section{Conclusion}

A resounding agreement occurred amount the reviewed studies that the inclusion of NPs in the manufacturing of titanium implants enhanced biocompatibility of the implants. Furthermore, majority of the studies showed 
that evaluating biocompatibility without exploring antimicrobial interventions might result in the benefits of NPs not being fully exploited. Thus, the combination of antibacterial ability and biocompatibility, as well as non-cytotoxicity, studied mainly in vitro indicates that the optimal nanoparticle enriching method could provide a promising strategy for the fabrication of long-term tissueimplant integration. As recommended by majority the authors included in this systematic review, future work on long-term implantation in vivo should be reported in future publications and a variety of nanoparticle materials should be explored.

Acknowledgment. The authors are grateful for the funding received from the Council for Scientific and Industrial Research (CSIR), the Collaborative Programme in Additive Manufacturing (CPAM) (Contract No: CSIR-NLC-CPAM-18-MOA-CUT01 ) and the Manufacturing, Engineering and Related Services Sector Education and Training Authority (merSETA). The author would like to thank Professor Annabel Fossey of the Central University of Technology, Free State, for providing the guidelines of structuring a systematic review.

\section{References}

1. P. Balakrishnan, M.S. Sreekala, S. Thomas, Fundamental Biomaterials: Metals (Woodhead Publishing, Duxford, 2018)

2. C. Oldani, A. Dominguez, Titanium as a biomaterial for implants, in Recent Advances in Arthroplasty, edited by S. Fokter (IntechOpen, London, 2012), pp. 149-162

3. J.-K. Du, C.-Y. Chao, K.-Y. Chiu, Y.-H. Chang, K.-K. Chen, J.-H. Wu, J.-N. Wu, Antibacterial properties and corrosion resistance of the newly developed biomaterial, $\mathrm{Ti}-12 \mathrm{Nb}-1 \mathrm{Ag}$ alloy, Metals 7 (2017) 566

4. M. Jäger, H.P. Jennissen, F. Dittrich, A. Fischer, H.L. Köhling, Antimicrobial and osseointegration properties of nanostructured titanium orthopaedic implants, Materials 10 (2017) 1302

5. M. Ziąbka, E. Menaszek, J. Tarasiuk, S. Wronski, Biocompatible nanocomposite implant with silver nanoparticles for otology-in vivo evaluation, Nanomaterials 8 (2018) 764

6. J.A. García, P.J. Rivero, R. Ortiz, I. Quintana, R.J. Rodríguez, Advanced surface treatments for improving the biocompatibility of prosthesis and medical implants, in Advanced Surface Engineering Research, edited by M.A. Chowdhury (IntechOpen, London, 2018), pp. 199-219

7. W. Paul, C.P. Sharma, Inorganic nanoparticles for targeted drug delivery, in Biointegration of Medical Implant Materials, edited by C.P. Sharma (Woodhead Publishing, Cambridge, 2020), pp. 333-373

8. V. Dahiya, P. Shukla, S. Gupta, Surface topography of dental implants: a review, J. Dental Implants 4 (2014) 66-71

9. F. Parnia, J. Yazdani, V. Javaherzadeh, S. Maleki Dizaj, Overview of nanoparticle coating of dental implants for enhanced osseointegration and antimicrobial purposes, J. Pharm. Pharm. Sci. 20 (2017) 148-160

10. G.G. Walmsley, A. McArdle, R. Tevlin, A. Momeni, D. Atashroo, M.S. Hu, A.H. Feroze et al., Nanotechnology in bone tissue engineering, Nanomedicine 11 (2015) 1253-1263
11. A. Radtke, M. Grodzicka, M. Ehlert, T. Jedrzejewski, M. Wypij, P. Golinska, To be microbiocidal and not to be cytotoxic at the same time... - silver nanoparticles and their main role on the surface of titanium alloy implants, J. Clin. Med. 8 (2019) 334

12. D. Beutner, K.-B. Hüttenbrink, Passive and active middle ear implants, GMS Curr. Topics Otorhinolaryngol. Head Neck Surg 8 (2009) Doc09

13. N. Karak, Fundamentals of sustainable nanostructural materials at bio-nano interface, in Dynamics of Advanced Sustainable Nanomaterials and Their Related Nanocomposites at the Bio-nano Interface, edited by N. Karak (Elsevier, St. Louis, 2019), pp. 1-24

14. A.M. Methley, S. Campbell, C. Chew-Graham, R. McNally, S. Cheraghi-Sohi, PICO, PICOS and SPIDER: a comparison study of specificity and sensitivity in three search tools for qualitative systematic reviews, BMC Health Serv. Res. 14 (2014) 579

15. D. Moher, A. Liberati, J. Tetzlaff, D.G. Altman, The PRISMA Group, Preferred reporting items for systematic reviews and meta-analyses: the PRISMA statement, PLoS Med. 6 (2009) e1000097

16. J.P. Higgins, D.G. Altman, P.C. Gøtzsche, P. Jüni, D. Moher, A.D. Oxman, J. Savovic et al., The Cochrane collaboration's tool for assessing risk of bias in randomised trials, BMJ 343 (2011) d5928

17. I. Lampé, D. Beke, S. Biri, I. Csarnovics, A. Csik, Z. Dombrádi, P. Hajdu et al., Investigation of silver nanoparticles on titanium surface created by ion implantation technology, Int. J. Nanomed. 14 (2019) 4709-4721

18. M. Dziaduszewska, M. Wekwejt, M. Bartmanski, A. Pałubicka, G. Gajowiec, T. Seramak, A.M. Osyczka, A. Zielinski, The effect of surface modification of Ti13Zr13Nb alloy on adhesion of antibiotic and nanosilver-loaded bone cement coatings dedicated for application as spacers, Materials 12 (2019) 2964

19. Y. Yin, Y. Li, W. Cai, J. Sui, One-step deposition of antibacterial Ag@Pdop hybrid films on an NiTi alloy, RSC Adv. 9 (2019) 29263-29272

20. J. Wang, G. Wu, X. Liu, G. Sun, D. Li, H. Wei, A decomposable silica-based antibacterial coating for percutaneous titanium implant, Int. J. Nanomed. 12 (2017) 371-379

21. E. De Giglio, D. Cafagna, S. Cometa, A. Allegretta, A. Pedico, L.C. Giannossa, L. Sabbatini, An innovative, easily fabricated, silver nanoparticle-based titanium implant coating: development and analytical characterization, Anal. Bioanal. Chem. 405 (2013) 805-816

22. H. Zhang, M. Hatoko, D. Yin, Y. Yang, Y. Zeng, S. Komasa, T. Kusumoto et al., Antibacterial activity and biocompatibility of nanoporous titanium doped with silver nanoparticles and coated with N-acetyl cysteine, J. Hard Tissue Biol. 27 (2018) 351-358

23. A.S.K. Kiran, T.S. Kumar, R. Sanghavi, M. Doble, S. Ramakrishna, Antibacterial and bioactive surface modifications of titanium implants by $\mathrm{PCL} / \mathrm{TiO}_{2}$ nanocomposite coatings, Nanomaterials 8 (2018) 860

24. Y. Yang, Y. Zhang, R. Hu, Q. Huang, K. Wu, L. Zhang, P. Tang, C. Lin, Antibacterial and cytocompatible AgNPs constructed with the assistance of Mefp-1 for orthopaedic implants, RSC Adv. 7 (2017) 38434-38443

25. J. Liao, M. Anchun, Z. Zhu, Y. Quan, Antibacterial titanium plate deposited by silver nanoparticles exhibits cell compatibility, Int. J. Nanomed. 5 (2010) 337-342 
26. S. Ferraris, A. Venturello, M. Miola, A. Cochis, L. Rimondini, S. Spriano, Antibacterial and bioactive nanostructured titanium surfaces for bone integration, Appl. Surf. Sci. 311 (2014) 279-291

27. S. Kranz, A. Guellmar, A. Voelpel, T. Lesser, S. TonndorfMartini, J. Schmidt, C. Schrader et al., Bactericidal and biocompatible properties of plasma chemical oxidized titanium $\left(\mathrm{TiOB}^{\circledR}\right)$ with antimicrobial surface functionalization, Materials 12 (2019) 866

28. I.S.V. Marques, M.F. Alfaro, M.T. Saito, N.C. da Cruz, C. Takoudis, R. Landers, M.F. Mesquita et al., Biomimetic coatings enhance tribocorrosion behavior and cell responses of commercially pure titanium surfaces, Biointerphases $\mathbf{1 1}$ (2016) 031008

29. S. Vignesh Nayar, Bhuminathan, Mahadevan, S. Santhosh, Comparative evaluation of the three different surface treatments - conventional, laser and nano technology methods in enhancing the surface characteristics of commercially pure titanium discs and their effects on cell adhesion: an in vitro study, J. Pharm. Bioallied Sci. 7 (2015) S87-S91

30. Z. Wang, Y. Sun, D. Wang, H. Liu, R.I. Boughton, In situ fabrication of silver nanoparticle-filled hydrogen titanate nanotube layer on metallic titanium surface for bacteriostatic and biocompatible implantation, Int. J. Nanomed. 8 (2013) 2903-2916

31. M. Kaczmarek, K. Jurczyk, J.K. Koper, A. Paszel-Jaworska, A. Romaniuk, N. Lipinska, J. Zurawski, P. Urbaniak, J. Jakubowicz, M.U. Jurczyk, In vitro biocompatibility of anodized titanium with deposited silver nanodendrites, J. Mater. Sci. 51 (2016) 5259-5270

32. M. Guan, Y. Chen, Y. Wei, H. Song, C. Gao, H. Cheng, Y. Li, K. Huo, J. Fu, W. Xiong, Long-lasting bactericidal activity through selective physical puncture and controlled ions release of polydopamine and silver nanoparticles-loaded $\mathrm{TiO} 2$ nanorods in vitro and in vivo, Int. J. Nanomed. 14 (2019) 2903-2914

33. A. Shivaram, S. Bose, A. Bandyopadhyay, Mechanical degradation of $\mathrm{TiO}_{2}$ nanotubes with and without nanoparticulate silver coating, J. Mech. Behavior of Biomed. Mater. 59 (2016) 508-518

34. X. Lu, B. Zhang, Y. Wang, X. Zhou, J. Weng, S. Qu, B. Feng, F. Watari, Y. Ding, Y. Leng, Nano-Ag-loaded hydroxyapatite coatings on titanium surfaces by electrochemical deposition, J. Roy. Soc. Interf. 8 (2011) 529-539

35. X. Zeng, S. Xiong, S. Zhuo, C. Liu, J. Miao, D. Liu, H. Wang, Y. Zhang, Z. Zheng, Nanosilver/poly (DL-lactic-co-glycolic Acid) on titanium implant surfaces for the enhancement of antibacterial properties and osteoinductivity, Int. J. Nanomed. 14 (2019) 1849-1863

36. W. Zhang, S. Wang, S. Ge, J. Chen, P. Ji, The relationship between substrate morphology and biological performances of nano-silver-loaded dopamine coatings on titanium surfaces, Royal Soc. Open Sci. 5 (2018) 172310

37. M. Akter, M.T. Sikder, M.M. Rahman, A.K.M.A. Ullah, K.F. B. Hossain, S. Banik, T. Hosokawa, T. Saito, M. Kurasaki, A systematic review on silver nanoparticles-induced cytotoxicity: physicochemical properties and perspectives, J. Adv. Res. 9 (2018) 1-16

38. G. Sánchez-Sanhueza, D. Fuentes-Rodríguez, H. BelloToledo, Copper nanoparticles as potential antimicrobial agent in disinfecting root canals. a systematic review, Int. J. Odontostomatol. 10 (2016) 547-554

39. N.G. de Oliveira, P.R. de Souza Araújo, M.T. da Silveira, A. P. Veras Sobral, M.V. Carvalho, Comparison of the biocompatibility of calcium silicate-based materials to mineral trioxide aggregate: systematic review, Eur. J. Dent. 12 (2018) 317-326

Cite this article as: Nthabiseng Nhlapo, Thywill Cephas Dzogbewu, Olga de Smidt, A systematic review on improving the biocompatibility of titanium implants using nanoparticles, Manufacturing Rev. 7, 31 (2020) 